\title{
Efficacy and safety of propiverine in SCI-patients suffering from detrusor hyperreflexia - A double-blind, placebo-controlled clinical trial
}

\author{
M Stöhrer ${ }^{1}$, H Madersbacher*,2, R Richter ${ }^{3}, \mathrm{~J}^{*}$ Wehnert ${ }^{4}$ and K Dreikorn ${ }^{5}$ \\ ${ }^{1}$ Traumatological Hospital, Department of Urology, Murnau, Germany; ${ }^{2}$ University Hospital of Innsbruck, Austria; \\ ${ }^{3}$ Zentralklinik Bad Berka, Germany; ${ }^{4}$ Urologist, Dresden, Germany; ${ }^{5}$ St. Jürgens Hospital, Department of Urology, \\ Bremen, Germany
}

\begin{abstract}
Aims of the study: The aim of this double-blind, randomised, prospective, multicentre trial was to evaluate the efficacy of propiverine in patients suffering from detrusor hyperreflexia caused by spinal cord injury in comparison to placebo.

Study design: The treatment period of 14 days comprised visits at baseline (V1) and after 14 days treatment (V2). Fifteen $\mathrm{mg}$ propiverine t.i.d. or placebo t.i.d. were administered as medication. The following efficacy parameters were adopted: the urodynamic parameters maximal cystometric bladder capacity, bladder volume on onset of the first as well as duration and amplitude of the maximum detrusor contraction, bladder compliance and residual urine, and subjective assessment of efficacy by physicians. For the evaluation of the safety of propiverine the incidence rate of adverse events by directly questioning as well as laboratory parameters were investigated. For biometrical evaluation $t$-test for independent groups was applied.

Results: One hundred and thirteen patients were investigated. The maximal cystometric bladder capacity increased significantly in the propiverine group, on average by $104 \mathrm{ml}$ (V1: $262 \pm 132 \mathrm{ml}$. V2: $366 \pm 143 \mathrm{ml}, P<0.001)$. The changes in bladder capacity during the first contraction and the maximum detrusor contraction in the verum group were both statistically significant. The bladder compliance documented a more pronounced increase under propiverine in comparison to placebo. Residual urine increased by $37 \pm 71 \mathrm{ml}$ in the propiverine group, significantly more than in the placebo group $(P=0.01)$. Sixty-three per cent of the patients expressed subjectively an improvement under propiverine in comparison with $23 \%$ of the placebo group. Expected anticholinergic adverse events occurred: dryness of the mouth (37\% in the verum and $8 \%$ in the placebo group), accommodation disorders $(28 \%$ and $2 \%$ respectively). Nausea, constipation, headache, dizziness, tiredness and palpitations were reported in almost comparable incidence rates between 3 and 13\% in both treatment groups.

Eight drop-outs were registered in the propiverine group (five due to adverse events) and three in the placebo group (one due to adverse events). The laboratory parameters revealed no changes.

Conclusion: Propiverine proved its efficacy in detrusor hyperreflexia with regard to the urodynamic parameters of the maximal cystometric bladder capacity and detrusor contractility. Anticholinergic adverse events such as dryness of the mouth and accommodation disorders were considered being tolerable. The increase in residual urine reflects the therapeutically desired effect of detrusor relaxation because the majority of patients normally practise intermittent catheterisation for bladder emptying.
\end{abstract}

Keywords: detrusor hyperreflexia; spinal cord injury; propiverine; urodynamics

\section{Introduction}

Propiverine hydrochloride (propiverine) is a spasmolytic agent (Apogepha, pharmaceutical factory, Dresden, Germany). Due to its dual mode of action-antic-

*Correspondence: $\operatorname{Dr} \mathrm{H}$ Madersbacher, University Hospital of Innsbruck, Annichstrasse 35, A-6020, Innsbruck, Austria holinergic and calcium-antagonistic effect on the smooth muscles-propiverine should have a powerful effect on the hyperreflexive detrusor.

The pharmacologic profile of propiverine has been extensively studied ${ }^{5,16}$. In vitro and in vivo studies revealed competitive anticholinergic properties of 
propiverine in comparison to atropine and oxybutynin, the calcium-antagonism is documented in several studies, eg in comparison to nifedipine. ${ }^{1-3}$ In numerous clinical trials propiverine has proved to be an effective drug in urinary incontinence due to detrusor overactivity with a moderate incidence of adverse events. $^{4-7}$

Following dose optimizing clinical trials, ${ }^{7}$ the aim of this study was the proof of efficacy and safety of propiverine in patients suffering from detrusor hyperreflexia in comparison to placebo.

\section{Materials and methods}

One hundred and thirteen patients were randomised to the propiverine group (60 patients) or to the placebo group (53 patients). Inclusion criteria were in-patients over 18 years of age suffering from detrusor hyperreflexia and suprasacral spinal cord injury. Exclusion criteria were pregnancy, cardiac, hepatic and renal dysfunctions, if requiring medical intervention, intestinal and urogenital obstructions, narrow angle glaucoma, severe psychotics and acute infections of the urinary tract.

In this double-blind, randomised, prospective, multicenter clinical trial treatment results of propiverine and placebo were compared in a parallel group design. The study was performed in accordance with the Declaration of Helsinki and Good Clinical Practice Guidelines. Patient's informed consent was given. Fifteen $\mathrm{mg}$ propiverine (sugar-coated tablets, registered as Mictonorm ${ }^{\mathbb{R}}$ in Germany or Detrunorm ${ }^{\mathbb{R}}$ in other European countries; manufactured and supplied by Apogepha Arzneimittel GmbH, Dresden, Germany) or sugar coated placebo tablets were administered t.i.d. for 14 days. Apogepha supported the study by an educational grant.

Evaluation of efficacy and safety comprised two visits (V1-day 0, V2-day 14):

(1) urodynamic parameters: maximal cystometric bladder capacity, bladder capacity during onset of the first detrusor contraction, duration and amplitude of the maximal detrusor contraction, bladder compliance and residual urine according to ICS criteria;

(2) patients' clinical symptoms assessed by a 3-point rating scale: improved, unchanged or worsened (V2);

(3) physicians' assessment of efficacy by a 3-point rating scale: very good or good, satisfactory or insufficient (V2);

(4) the incidence rates of adverse events by directly questioning the patients vital parameters (blood pressure, heart rate) (V1, V2);

(5) laboratory parameters (haematology: ESR, erythrocyte count and shape, leukocyte count, thrombocyte and reticulocyte count, differential blood count, Hb, HCT, PTT, QUICK's method, bleeding time; clinical chemistry: AP, GOT, GPT, bilirubin, $\mathrm{Na}, \mathrm{K}, \mathrm{Ca}$, chloride, phosphatase, cholesterol, cholinesterase, triglyceride, total pro- tein, glucose, uric acid, creatinine; urinalysis: blood, glucose, ketone bodies, $\mathrm{pH}$, sediment, urobilinogen) (V1, V2).

For biometrical evaluation $t$-test for independent groups was applied.

\section{Results}

Four study centres recruited 113 patients (69 men and 44 women). Demographic data and patients' history of the two parallel groups demonstrated no statistical differences: in the propiverine group 37 male and 23 female patients with a mean age of $30.3 \pm 11.7$ years, and in the placebo group 32 male and 21 female patients with an average age of $29.3 \pm 10.9$ years were included.

\section{Efficacy}

The bladder capacity on onset of the first detrusor contraction increased statistically significant by $72 \mathrm{ml}$ under propiverine (V1: $153 \pm 107 \mathrm{ml}$. V2: 224 $\pm 111 \mathrm{ml}$ ) in contrast to $35 \mathrm{ml}$ under placebo (V1: $147 \pm 95 \mathrm{ml}$. V2: $159 \pm 107 \mathrm{ml})(P=0.006)$.

The maximal cystometric bladder capacity increased significantly in the propiverine group, on average by $104 \mathrm{ml} \quad(\mathrm{V} 1: \quad 262 \pm 132 \mathrm{ml} ; \quad \mathrm{V} 2: \quad 366 \pm 143 \mathrm{ml}$ ) $(P<0.0001)$, in comparison with a decrease under placebo (V1: 296 $\pm 151 \mathrm{ml}$; V2: $289 \pm 163 \mathrm{ml}$ ) (Figure 1).

The maximal detrusor contractions decreased significantly by $27 \pm 32 \mathrm{cmH}_{2} \mathrm{O}$ (V1: $80.7 \pm 40.3$; V2: $\left.53.6 \pm 32.2 \mathrm{cmH}_{2} \mathrm{O}\right) \quad(P<0.001)$ under propiverine, reaching significance as well in comparison with placebo (V1: $91.7 \pm 37.2 ; \quad$ V2: $\left.91.9 \pm 46.2 \mathrm{cmH}_{2} \mathrm{O}\right)$ $(P<0.0001)$ (Figure 2).

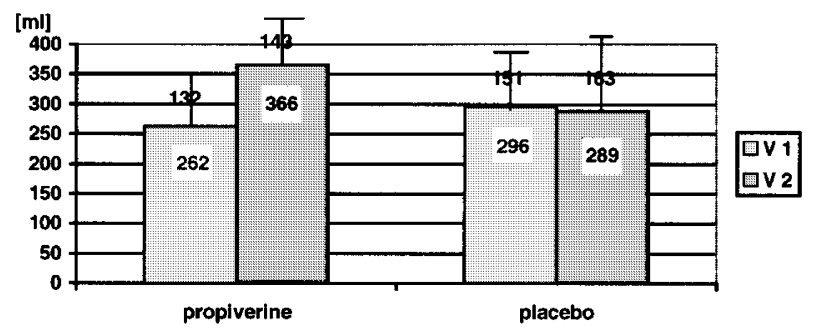

Figure 1 Maximal cystometric bladder capacity

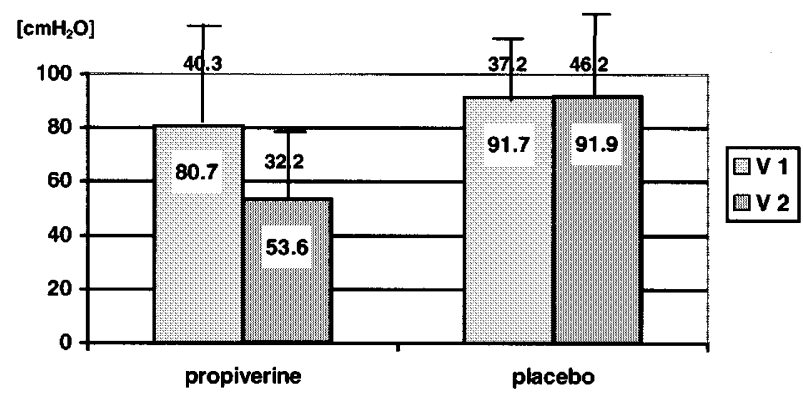

Figure 2 Maximal detrusor contractions 
The duration of the detrusor contractions decreased by $20.0 \mathrm{~s}$ (V1: $57.9 \pm 53.7: \mathrm{V} 2: 37.9 \pm 38.4 \mathrm{~s}$ ) under propiverine and by $1.6 \mathrm{~s}$ (V1: $46.6 \pm 32.5$; V2: $45.0 \pm 33.6 \mathrm{~s})$ under placebo demonstrating a significant difference between both treatment groups $(P=0.03)$.

The compliance (detrusor coefficient) documented a more pronounced increase under propiverine $(\mathrm{V} 1$ : $16.6 \pm 12.9$; V2: $21.8 \pm 15.8 \mathrm{ml} / \mathrm{cmH}_{2} \mathrm{O}$ ) in comparison to placebo (V1: $15.2 \pm 10.9 ; \quad \mathrm{V} 2: \quad 17.2 \pm 11.9 \mathrm{ml} /$ $\mathrm{cmH}_{2} \mathrm{O}$ ). However, significance could not be demonstrated.

Residual urine increased by $36.8 \pm 71 \mathrm{ml}$ (V1: $49.7 \pm 109.4 ; \mathrm{V} 2: 86.5 \pm 109.3 \mathrm{ml})$ in the propiverine group only. This increase was significant $(P=0.01)$ in comparison with the placebo group (V1: $58.9 \pm 90.2$; V2: $60.8 \pm 71.9 \mathrm{ml})$. Sixty-one $(53.9 \%)$ of the patients documented the mode of bladder emptying which did not vary between treatment groups and during treatment. Without considering missing data $86.9 \%$ of the patients voided spontaneously or by triggering, $13.1 \%$ of the patients practised intermittent catheterisation.

The clinical symptoms assessed by the patients revealed that $63.3 \%$ under propiverine were 'improved' in comparison with $22.6 \%$ under placebo. 'Unchanged' symptoms resulted in $16.7 \%$ of the patients under verum, in $56.6 \%$ under placebo. 'Worsening' of symptoms was assessed in $16 \%$ of the patients in both of the treatment groups. This assessment is based on the micturition diaries concerning clinical symptoms: the reduction of micturition frequency, enhancement of dry intervals between catheterisations and the achievement of urinary continence, respectively.

Subjective global assessment of efficacy by physicians was 'very good' or 'good' in $53 \%$ of the patients under propiverine but only in $11 \%$ of the patients under placebo. In addition, a 'satisfactory' efficacy was certified in $25 \%$ of the verum group in contrast to $17 \%$ of the placebo group. 'Insufficient' treatment resulted in $18 \%$ under propiverine but in $64 \%$ under placebo.

\section{Safety}

Direct questioning of adverse events yielded incidence rates between 3 and $37 \%$ (Table 1). Twenty-two out of

Table 1 Incidence rates of adverse events

\begin{tabular}{lcc}
\hline & $\begin{array}{c}\text { Propiverine } \\
\%\end{array}$ & $\begin{array}{c}\text { Placebo } \\
\%\end{array}$ \\
\hline Dryness of the mouth & 37 & 8 \\
Accommodation disorders & 28 & 2 \\
Nausea & 5 & 9 \\
Constipation & 3 & 6 \\
Headache & 10 & 8 \\
Dizziness & 10 & 4 \\
Tiredness & 13 & 4 \\
Palpitations of the heart & 10 & 8 \\
\hline
\end{tabular}

60 patients in the verum group $(37 \%)$ and four out of 53 patients in the placebo group $(8 \%)$ complained of dryness of the mouth. In most of the cases the severity was judged to be mild or moderate under verum as well as under placebo (Figure 3).

The accommodation disorders were documented as mild in nine, moderate in seven and severe in one case under propiverine, mild in the case of placebo. Nausea, headache, dizziness, tiredness and heart palpitations were reported to be mild or moderate. The vital parameters (blood pressure and heart rate) and the laboratory parameters revealed no changes regarding the comparison of both groups.

Eight drop-outs were registered in the propiverine group (13\%; five due to adverse events, three cases without mentioning the underlying reasons) and three in the placebo group $(7.5 \%$; one due to adverse events, two cases without mentioning the underlying reasons).

\section{Discussion}

The aims of treatment of detrusor hyperreflexia are the preservation of kidney function and the control of reflex urinary incontinence. Preservation of kidney function is surely of prime interest and guarantees a life expectancy almost comparable to non spinal cord injured people. In the presence of detrusor hyperreflexia micturition can be achieved either spontaneously once the urine in the bladder reaches reflex volume or by trigger mechanisms. Reflex voiding is achieved via a sacral reflex arch through C-fibre activation. This mostly induces a detrusor-striated sphincter-dyssynergia resulting in unbalanced voiding and with the potential danger for the upper urinary tract. The bladder contractions themselves are not sustained and may be hypercontractile but also hypocontractilebeing an additional reason for unbalanced voiding. Triggered reflex micturition can only be recommended if the contractility of the detrusor is within normal ranges, if the bladder compliance is normal and bladder emptying balanced. About $25 \%$ of patients fulfil these criteria. However, with unphysiological high pressures during the filling phase (low compliance) and during the micturition phase, with recurrent urinary

[n]

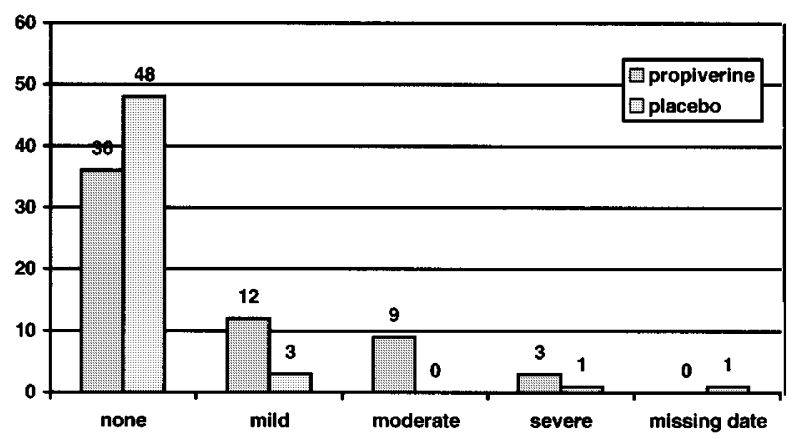

Figure 3 Severity of the dryness of the mouth (V2) 
tract infections caused by unbalanced voiding, with vesico-ureteral-renal reflux or reflux into the male adnexe due to a pronounced detrusor-striated sphincter-dyssynergia treatment of detrusor hyper-reflexia is mandatory. The therapeutic standard now-adays is urinary bladder emptying by (clean) intermittent catheterisation (CIC) and pharmacological treatment of a low compliant and hypercontractile detrusor in order to change the high pressure into a low pressure system. The new generation of smooth muscle relaxant drugs with mixed mode of action: anticholinergic, direct spasmolytic, calcium-channel blocking and local anaesthetic properties, guarantees that hyperreflexia of the detrusor can be well controlled in the majority of patients, thus preserving the upper urinary tract and enabling continence. ${ }^{9}$

The simultaneous increase of residual urine is well accepted in these patients normally emptying their bladder by CIC and in whom the pharmacologically induced detrusor hypocontractility guarantees continence between catherisations. As demonstrated by Yokota et al, ${ }^{10}$ anticholinergics are especially effective in the reflex bladder as the contribution of cholinergic transmission to detrusor contraction is considerably increased, whereas the purinergic component is decreased in comparison with normals. One must assume that the postganglionic neurotransmission in the reflex bladder may be shifted to a cholinergic dominance. This might explain that the effect of these drugs is much more pronounced in detrusor hyperreflexia in comparison to detrusor instability.

Propiverine possesses strong calcium-antagonistic and moderate anticholinergic-spasmolytic properties as well as analgesic effects. Considering the pharmacological profile of propiverine, it might be considered as a special advantage that metabolites with a strong anticholinergic mode of action contribute further to the effects of the parent drug. ${ }^{2,3,11}$ Increase in maximum cystometric bladder capacity by $30 \%$ as well as the decrease of detrusor contractility by more than $40 \%$ are comparable to the results achieved with oxybutynin ( $5 \mathrm{mg}$ t.i.d.) as published by Madersbacher et al. $^{12}$ The dosage regimen selected according to the results of a previous dose optimizing study ${ }^{7}$ was confirmed for this indication. The increase in residual urine is comparable to that of oxybutynin, which currently is considered to be the therapeutic standard and which is one of the most commonly used drugs, world-wide accepted in the treatment of detrusor hyperreflexia. The urodynamic results further document that propiverine also reduces effectively detrusor pressure.

It is generally accepted that with the increase of effectiveness of these drugs, the rate of adverse events also increases. This is also true for dryness of the mouth, one of the most frequent and most bothersome adverse events, manifesting under oxybutynin: Thüroff et $a l^{13}$ reported $33 \%$ of patients with detrusor hyperactivity treated with oxybutynin complaining about dryness of the mouth. Madersbacher et al reported an incidence of $57 \%$ in patients with spinal cord injury and detrusur hyperreflexia treated with oxybutynin $5 \mathrm{mg}$ t.i.d. In $23 \%$ this symptom was severe and one of the main reasons for drop-outs. Under propiverine the incidence of dryness of the mouth as well as other adverse events shows a dose dependency as well as a decreasing incidence under long-term treatment. ${ }^{7,14}$ In the present study, the rate of dryness of the mouth is somewhat higher than in spontaneous reports, but lower than in studies with oxybutynin in comparable patient groups. This is in accordance with earlier studies reporting incidence rates of adverse events, especially dryness of the mouth, varying between $19 \%$ and $21 \%$ under propiverine. The severity was judged in most cases as mild or moderate yielding in a good or very good subjective assessment of tolerability in $61 \%$ to $81 \%$ of the patients. ${ }^{5}$

This clinical study proved the efficacy of propiverine in detrusor hyperreflexia with regard to the urodynamic parameters of maximal cystometric bladder capacity and detrusor contractility. Increase in residual urine reflects the therapeutically desired effect of detrusor relaxation in this special group of patients. Propiverine proved to be an effective and safe drug in the treatment of detrusor hyperreflexia in spinal cord injured patients.

\section{References}

1 Alloussi $\mathrm{S}$ et al. Effect of trospium chloride, oxybutynin and propiverine on the interaction of acetylcholine with isolated preparations of the human urinary bladder. Investigative Urology 1991; 4: $157-161$.

2 Riotte J, Mutschler E. Untersuchungen zur spasmolytischen Aktivität von Propiverin und einigen seiner Strukturanaloga. Arzneimittel-Forsch 1987; 37: 300-302.

3 Wada $\mathrm{Y}$ et al. Comparison of the effects of various anticholinergic drugs on human isolated urinary bladder. Arch Int Pharmacodyn 1995; 330: 76-89.

4 Halaska M, Dorschner W, Frank M. Treatment of urgency and incontinence in elderly patients with propiverine hydrochloride. Neurourology and Urodynamics 1994; 13: 428-430.

5 Mazur D et al. Klinische und urodynamische Effekte einer oralen Propiverintherapie bei neurogener Harninkontinenz. Urologe [A] 1994; 33: $447-452$.

6 Wehnert J, Sage S. Therapie der Blaseninstabilität und UrgeInkontinenz mit Propiverin hydrochlorid (Mictonorm ${ }^{\circledR}$ ) und Oxybutynin chlorid (Dridase ${ }^{\mathbb{R}}$ ) - eine randomisierte Cross-overVergleichsstudie. Akt Urol 1992; 23: 7-11.

7 Mazur D et al. Verträglichkeit und Wirksamkeit einer Langzeittherapie mit Propiverinhydrochlorid bei neurogener Harninkontinenz - Eine multizentrische Studie. Kontinenz 1994; 3: $74-78$.

8 Abrams P, Blaivas JG, Stanton SL, Andersen JT. The standardization of terminology of lower urinary tract function. Br J Obstet Gynaecol 1990; 97: suppl. 6, pp 1-16.

9 Knoll M, Madersbacher $\mathrm{H}$. The chances of a spina bifida patient becoming continent/socially dry by conservative therapy. Paraplegia 1993; 31: $22-27$.

10 Yokota T, Yamaguchi O. Changes in cholinergic and purinergic neurotransmission in pathologic bladder of chronic spinal rabbit. J Urol 1996; 156: 1862 - 1866.

11 Thümmler D. Human pharmacokinetics of propiverine hydrochloride (Mictonorm ${ }^{\circledR}$, Mictonetten ${ }^{\circledR}$, BUP- $4^{\circledR}$ tablet 10.20). Unpublished report February 1997. 
12 Madersbacher $\mathrm{H}$ et al. Trospium chloride versus oxybutinin: a randomized, double-blind, multicentre trial in the treatment of detrusor hyperreflexia. Br J Urol 1995; 75: 452-456.

13 Thüroff JW et al. Randomized, double-blind, multicenter trial on treatment of frequency, urgency and incontinence related to detrusor hyperactivity: oxybutynin versus propantheline versus placebo. J Urol 1991; 145: 813-817.

14 Madersbacher $\mathrm{H}$ et al. Tolerability and efficacy of propiverine hydrochloride in patients with urge incontinence in comparison to oxybutynin and placebo - a urodynamically controlled multicenter study. Br J Urol 1998; submitted.
15 Meisel P, Langner S, Siegmund W. In-vitro binding of propiverine hydrochloride and some of its metabolites to serum albumin in man. J Pharmacy a Pharmacol 1997; 33: 270-272.

16 Potthast H, Schug B, Blume H. Bioavailability study of Mictonorm Dragees (Apogepha Arzneimittel $\mathrm{GmbH}$ ) after single dose administration in comparison to multiple dose administration. Unpublished report, 1996. 